\title{
Implementation of Centralized Cooperative Spectrum Sensing Based on USRP
}

\author{
Yutang Fu \\ Information and Communication Engineering College \\ Harbin Engineering University \\ Harbin, China \\ e-mail: fuyuatng1988@gmail.com
}

\author{
Zhigang Li \\ Information and Communication Engineering College \\ Harbin Engineering University \\ Harbin, China \\ e-mail: lizhigang@hrbeu.edu.cn
}

\author{
Dandan Liu \\ Electronics and Information Engineering College \\ Heilongjiang Technology University \\ Harbin, China \\ e-mail: $512738371 @ q q . c o m$ \\ Qianli Liu \\ Information and Communication Engineering College \\ Harbin Engineering University \\ Harbin, China \\ e-mail: 798491372@qq.com
}

\begin{abstract}
In this paper, a system of centralized cooperative spectrum sensing is designed. Energy detection and cyclostationary feature detection are used by Secondary User. The stepping spectrum sensing function is used to sense and record the vacant spectrum around the location of $S U$ and then the information is uploaded to the control center with its coordinate. This system can make a pre-judgment for SU based on the uploaded information. And when have SU need to make a communication, the $\mathrm{SU}$ can find the vacant spectrum fast depending on the data fusion. In this system, we use NI-USRP2920, USRP1 as the hardware platform and GNU Radio, LabVIEW and HDSDR as the software platform.
\end{abstract}

Keywords-Energy Detection; USRP; Spectrum Sensing; Centralized Detection; GNU Radio

\section{INTRODUCTION}

Nowadays, we face the problem of radio spectrum resource shortage due to the rapid increase of wireless equipments and the traditional spectrum allocation strategy. The cognitive radio terminology was invented by Mitola, and refers to a smart radio which can sense the external RF environment, learn from the history and make intelligent decisions to adjust its transmission parameters according to the current state of the environment. As radio spectrum is the most important resource that need to be efficiently utilized, the cognitive radio technology has been proposed to fulfill this requirement of efficient radio spectrum utilization. Cognitive radio is very helpful in improving the use of spectrum resources and it can reduce the costs for people. In this system, coordinate of SU, vacant spectrum that with location and time information are stored in the database of the control center. The SU use energy detection and cyclostationary feature detection to get the vacant spectrum information. If SU want to have a communication, the control center will send the vacant spectrum information to the SU depending on its location and local time. And then frequency of operation, transmitter power and modulation and so on can automatically be adjusted in this CR system. This system can make a pre-judgment for SU according to the information of SU. At last, we use this system transmit a demo video to make a verification of how it works.

\section{SPECTRUM SENSING METHODS}

The present literature for spectrum sensing is still in its early stages of development. A number of different methods are applied for identifying the spectrum is vacant or not. But in most approaches, they just focus on the vacant radio spectrum sensing and ignore the data fusion. In this section, we use the two most common spectrum sensing techniques to sense the vacant spectrum, and then process the data fusion with the database in the control center. 


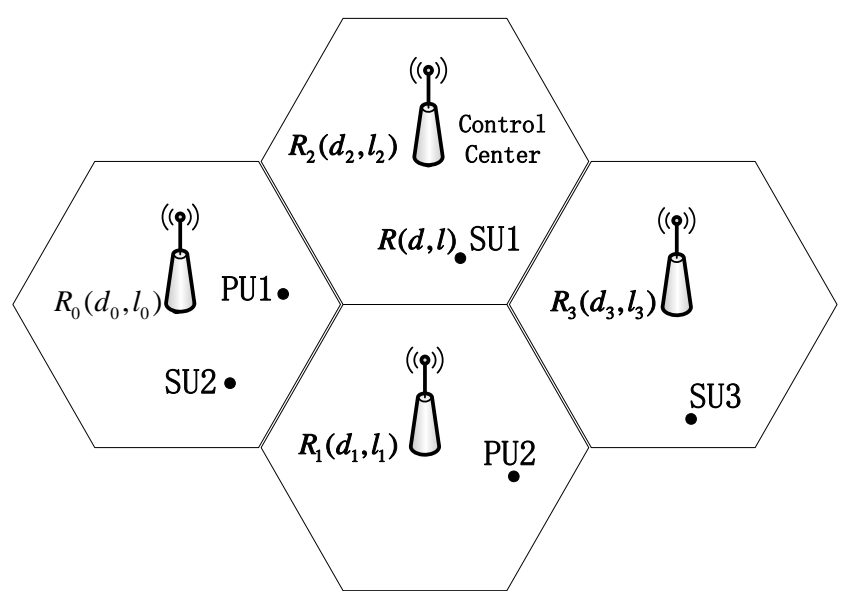

Figure 1. The placement of the element in CR system

In this system, considering the power limitation of the SU, we assume the CR control center placement like the Fig.1. Each control center and SU has a coordinate and stores in the database. The GPS (in this paper we assign addresses manually) provides the coordinate of SU, and SU will sense the vacant spectrum. These information is uploaded through the control channel. The SU nodes use energy detection and cyclostationary feature detection to realize the spectrum sensing.

\section{A. Energy Detection}

The detection problem for spectrum sensing at secondary user (SU) can be formulated as a binary hypothesis. $H_{0}$ corresponding to primary user (PU) signal absent, and the alternative hypothesis $H_{1}$ corresponding to PU signal present. Therefore, the goal of spectrum sensing is to decide between the following two hypotheses,

$$
\begin{aligned}
& H_{0}: x(t)=n(t) ; \\
& H_{1}: x(t)=s(t)+n(t) ;
\end{aligned}
$$

Where $0<t \leq T$, and $T$ denotes the observation time, $x(t)$ is the received signal by SU, $s(t)$ is the signal of the primary user. $n(t)$ is the AWGN that at any arbitrary time $t$ the value $n$ is a Gaussian random variable with zero mean and variance $\delta^{2}$. So the decision expression can be given as

$$
\bar{E}=\frac{1}{N} \sum_{1}^{N}|x(t)|_{\substack{H_{0} \\ \gtrless}}^{\underset{H_{1}}{\geq} \eta}
$$

In this expression, $\bar{E}$ is the decision variables of energy detection, $\eta$ is the threshold. When $\bar{E} \geq \eta$, the result is $H_{1}$, means that the PU exists, and the band is occupied otherwise the band is free.

\section{B. Cyclostationary Feature Detection}

Stationary signal usually via the transformation of sampling, modulation, coding, and pulse sequence and so on, and its statistical parameters have the feature of potential periodic. The detection method designed with this feature is called Cyclostationary Feature Detection. This detection method can get the symbol rate, modulation type and sinusoidal carrier and analyze the signal characteristics by spectral correlation function. Cyclic spectral density function of the modulated signal have a large discrete amplitude values in the non-zero loop frequency, but the stationary noise almost has no value or very small value in that frequency. So the PU signal and noise can be distinguished. And use cyclic spectral density $S(f ; \tau)$ to describe the detection standard.

The spectrum sensing model is given as

$$
S(f ; \tau)=\frac{1}{N} \sum_{n=1}^{N} y(n) y(n+\tau) e^{-j 2 \pi f n}
$$

We assume the signal received is

$$
y(n)=\sum_{k} s_{k}(n) \exp \left(j 2 \pi f_{k} n\right)+\omega(n)
$$

Where the $s_{k}(n)$ is the independent zero mean stationary process, $s_{k}(n)$ and Circularly Symmetric White Noise Sequence are independent of each other. So when $N \rightarrow \infty$, we can get the new $S(f ; \tau)$

$$
S(f ; \tau) \approx \sum_{k} R_{k}(\tau) e^{j 2 \pi f_{k} \tau} \delta\left(f-2 f_{k}\right)
$$

Where the $R_{k}(\tau)$ is

$$
R_{k}(\tau)=E\left[s_{k}(t) s_{k}(t+\tau)\right]
$$

This cycle feature detection methods can be easily achieved by FFT, but with the signal frequency jitter and nonlinear RF environment related.

From the Fig. 1 we can get the location function as

$$
\begin{aligned}
& S_{n}=\sqrt{\left(d-d_{n}\right)^{2}+\left(l-l_{n}\right)^{2}} \quad n \geq 0 \\
& S_{m}=\min \left\{S_{0}, S_{1}, S_{2}, S_{3} \cdots \cdots S_{n}\right\} \quad m \leq n
\end{aligned}
$$

The coordinates of the $\mathrm{SU}$ is $R(d, l)$ and Control Center is $R_{n}\left(d_{n}, l_{n}\right)$, so from the function (8) we can know the SU belongs to the $R_{m}$ area. Then the system make a decision by the statistic data.

In this system, we use USRP1 works as the SU and it has the additional function of spectrum sensing at free time for uploading. Fig. 2 shows the program processing of SU. 


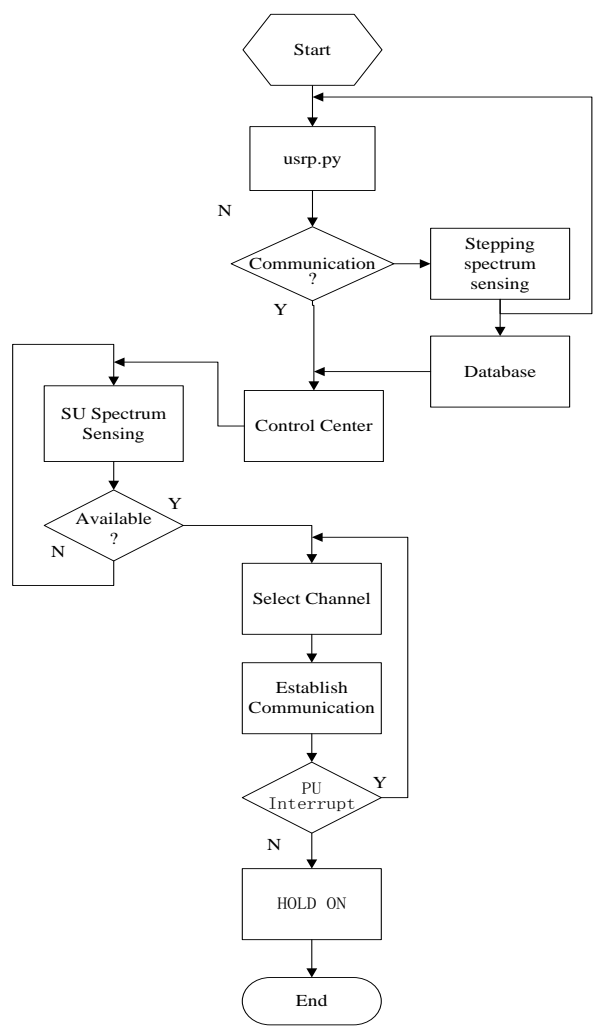

Figure 2. Program processing of SU

The program executes the usrp.py to initialize the usrp1, if it need to communicate, the message with coordinate will be sent to the control center through the control channel. And the control center will send order to SU for setting up the communication according to the data fusion. If the SU do not need to communicate, it will go on sensing the vacant spectrum using the cyclostationary feature detection, and then upload the local information with the coordinate, frequency, modulation and so on.

\section{EXPERIMENTATION PLATFORM}

As the Fig. 3 shows that the hardware of this system is NI-USRP2920 and USRP1. Software is GNU Radio, LabVIEW, HDSDR running on Windows XP and Ubuntu operating system. As it is shown that the USRP 2920 is the control center and the usrp1 is the SU and PU. SU make a communication with control center through the control channel, and the SU can set up a communication use the vacant spectrum.

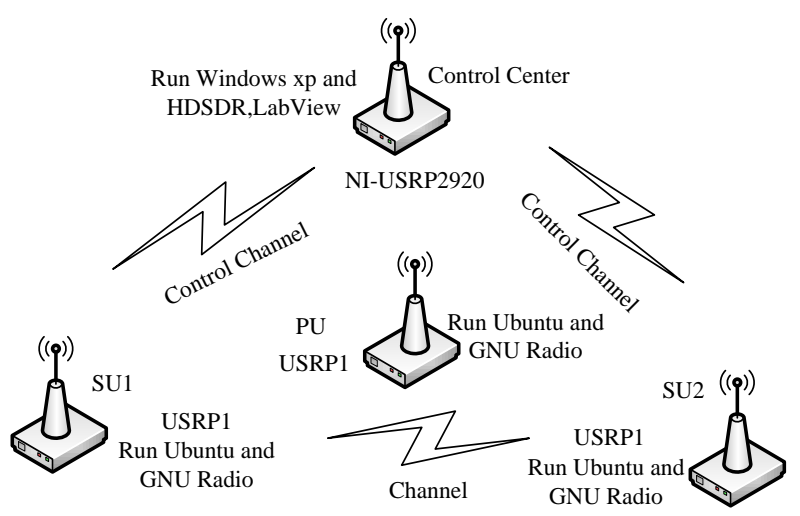

Figure 3. System components

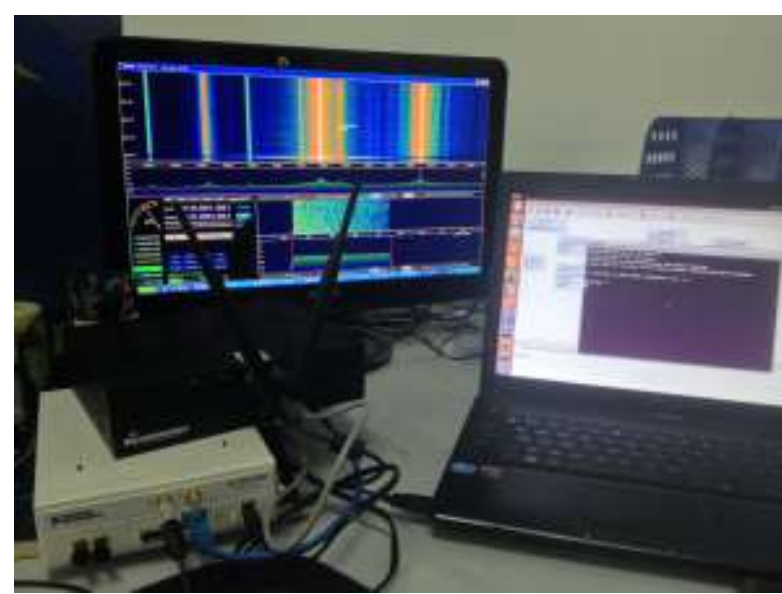

Figure 4. Experimental platform hardware

\section{Hardware Platform}

NI USRP-2920 software-programmable radio transceivers are designed for wireless communications teaching and research. Programmable with NI LabVIEW software, the USRP hardware is an affordable and easyto-use RF platform for rapid prototyping applications such as record \& playback, physical layer communication, spectrum monitoring and more. With the ability to transmit and receive RF signals across a wide range of frequencies from $50 \mathrm{MHz}$ to $2.2 \mathrm{GHz}$ with up to $40 \mathrm{MHz}$ of real-time bandwidth and plug-and-play MIMO support, the NI-USRP enables a broad range of communications applications covering common standards such as broadcast radio, digital TV, GSM Cellular, GPS, 802.11(WiFi) and ZigBee. LabView brings increased productivity with an intuitive graphical programming approach, and $\mathrm{m}$-file script compatibility enabling development of algorithms for physical layer communications [8]. 


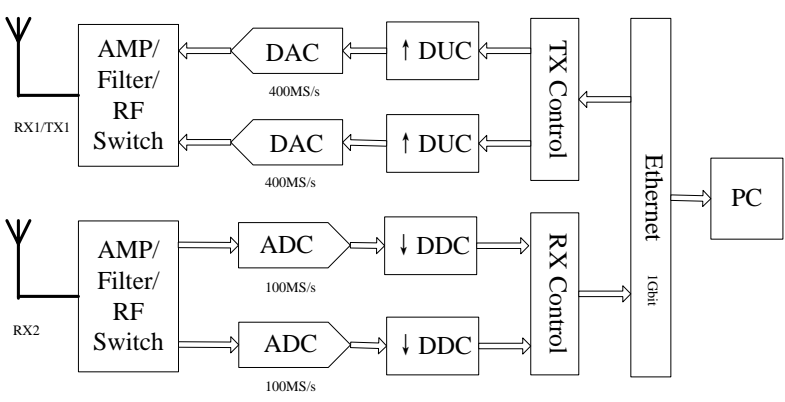

Figure 5. NI USRP-2920 System Block Diagram

Universal Software Radio Peripheral (USRP), a hardware platform for SDR , consists of FPGA, ADC/DAC, and USB controller as shown in Fig.6. At the transmitter and the receiver, the USRP motherboard is placed between the RF front-end and the host computer. FPGA is used to perform high bandwidth mathematical calculations such as decimation, modulation or demodulation, digital down conversion, digital up conversion and interpolation signal processing process. FPGA is also employed to reduce the data rate on spectrum channel to the data rate that can transmit over USB2.0 to the host computer. ADC/DAC converts the data format between analogue and digital. USB 2.0 is used to transmit and receive data between USRP and host computer for signal processing[5].

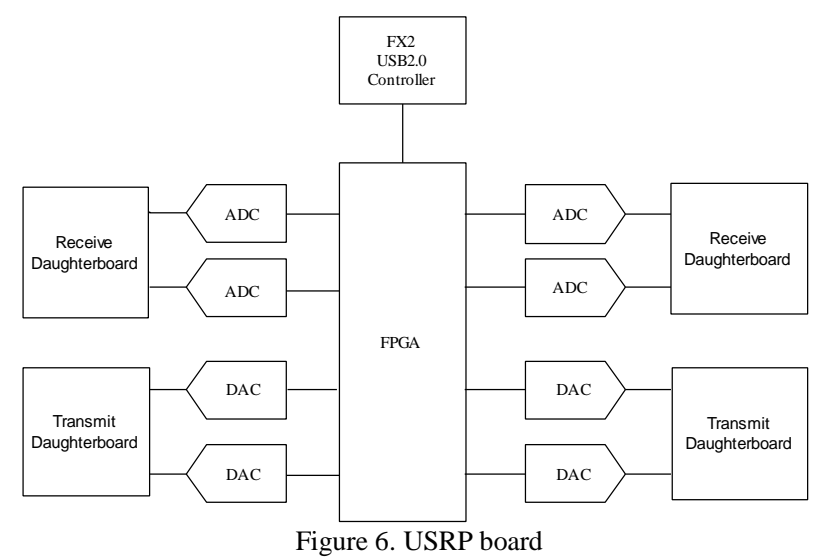

\section{B. Software Platform}

In this system, we use GNU Radio, LabVIEW and HDSDR as the Software Platform. GNU Radio is an open source software toolkit which consists of signal processing blocks library and the glue to tie these blocks together for building and deploying software defined radios are written in $\mathrm{C}++$ while python is used as a scripting language to tie the blocks together to form the flow graph. SWIG (Simplified Wrapper and Interface Generator)is use as the interface compiler which allows the integration between $\mathrm{C}++$ and Python language. GNU Radio practically implements the signal processing blocks on the general-purpose processor [9].

LabVIEW is a graphical programming platform that consists of signal processing and mathematical function library, and have more than one thousand signal processing, analysis, and math functions, including wavelet transform, time-frequency analysis, image processing, filter design, sound and vibration, system identification, RF analysis tools and other specialized methods of the package. We use it with the NI-USRP to process the signal received and make the data fusion. And as the control center, HDSDR is also running on the PC to monitor the RF spectrum. The HDSDR is connected with the IO driver and UHD through the USB interface. The Fig.7 shows the connection structure.

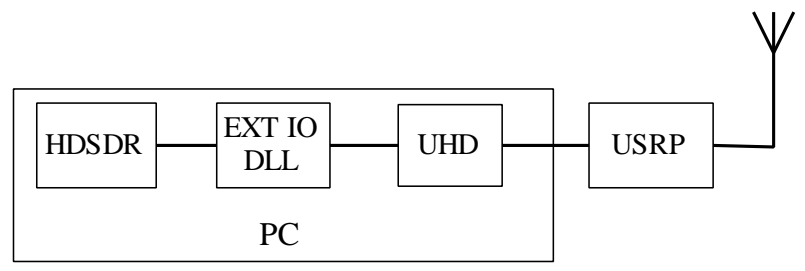

Figure 7. HDSDR connection structure

\section{CONCLUSION}

In this paper, we build a simple CR system to implement the centralized cooperative spectrum sensing and transmit a H.264 encoding video that formed by a picture to test it.

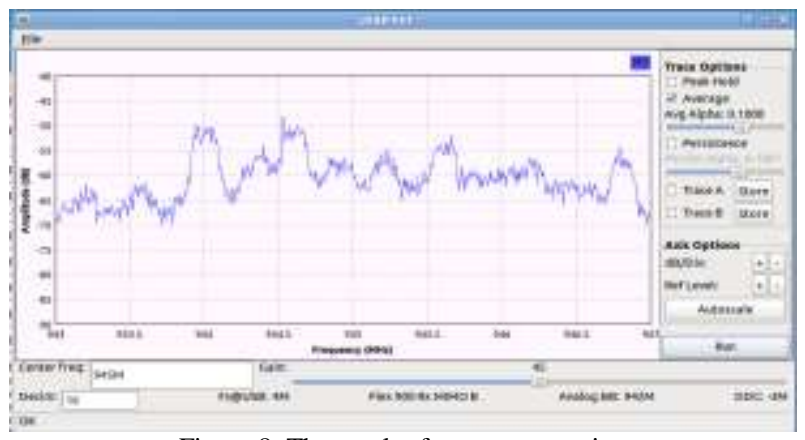

Figure 8. The result of spectrum sensing

Considering of the limitation of mobile devices in our real life, we use USRP1 as the SU. Because of bandwidth limitation of USRP1, it can only detect RF signal bandwidth of $8 \mathrm{MHz}$ once. So the SU need to make a sweep frequency by steeping the frequency of RF FrontEnd to sense a large bandwidth RF signal, and we set the stepping frequency $3 \mathrm{MHz}$. To evaluate the system presented, the video quality is measured as it is perceived at the receiving node. From the experiment result, we can see that the CR system using the centralized cooperative spectrum sensing have a better performance. The Fig.8 shows the monitor results of the frequency spectrum.

The SU clients set up communication depending on the data of control center. One SU runs tx_test.py and the other SU runs rx_test.py, then the SU will send the data to the control center. When the SU transmit the test_video.H264 file, the control center sent the command to set the communication parameters. Fig.9 shows the result of transmitting video, we can see the picture $B$ is damaged without the help of control center. Set up communication only by SU itself can not response 
frequency switching fast enough, and will cause mutual interference between SU and PU.

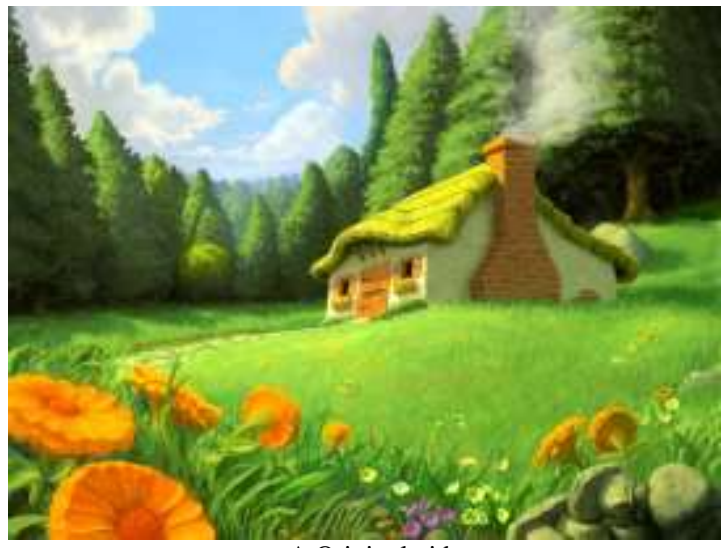

A Original video

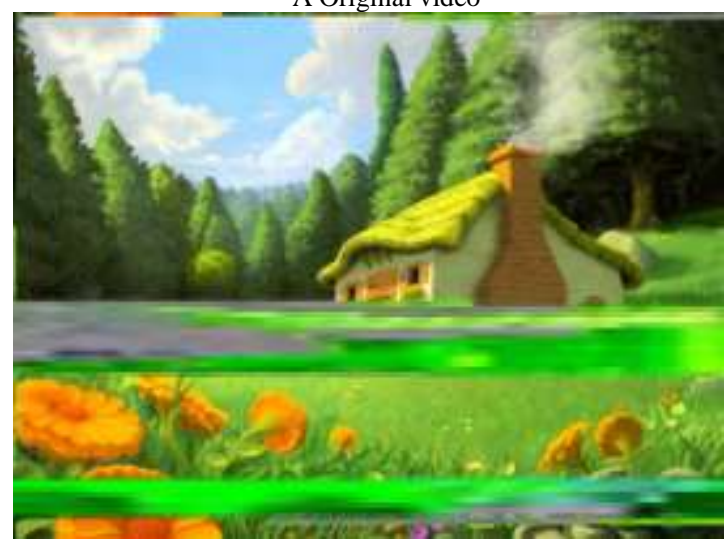

B Damaged video

Figure 9. The snapshots of testing result

\section{ACKNOWLEDGEMENT}

This paper is funded by the International Exchange Program of Harbin Engineering University for Innovationoriented Talents Cultivation and is supported by Natural Science Foundation of China. (51374099). Heilongjiang Province Natural Science Foundation for the Youth (No. QC2012C070), the Chinese University Scientific Fund (No. HEUCF130807).

\section{REFERENCES}

[1] Mohd Adib Sarijari, Arief Marwanto, Norsheila Fisal,et al, "Energy Detection Sensing based on GNU Radio and USRP: An analysis study," IEEE 9th Malaysia International Conference on Communications. IEEE Proc, Malaysia, pp.338-342. December 2009.

[2] Y. Zeng and Y. C. Liang, "Spectrum-sensing algorithms for cognitive radio based on statistical covariances," IEEE Transactions on Vehicular Technology, IEEE, pp.1804-1815. May 2009.

[3] Matt Ettus. USRP. http://www.ettus.com.

[4] Yu Zhang, Wen-dong Yang, Yue-ming Cai, "Cooperative spectrum sensing technique," IEEE International Conference on Communications Networking and Mobile Computing, IEEE Computer Society, Shanghai, China, pp.1167-1170. Sept. 2009.

[5] Z. Tong,M. S. Arifianto, C. F. Liau, "Wireless Transmission using Universal Software Radio Peripheral", 2009 International Conference on Space Science and Communication, IEEE Proc, Malaysia, pp.19-23. Dec.2009.
[6] Zhang, W, Mallik R K, Ben Letaief K. "Optimization of cooperative spectrum sensing with energy detection in cognitive radio networks." IEEE Transactions on Wireless Communications, IEEE, pp.5761-5766. Dec.2009.

[7] D. Cabric, S. Mishra, R. Brodersen, "Implementation issues in spectrum sensing for cognitive radios," Asilomar Conf. On Signals, Systems and Computers, IEEE Computer Society, California, USA, pp.772-776. Nov. 2004.

[8] National Instruments. NI USRP-29xx Data sheet. http://sine.ni.com/ds/app/doc/p/id/ds-355/lang/zhs.

[9] GnuRadio,http://gnuradio.org/redmine/projects/gnuradio.

[10] Mohammad Tahir, Hafizal Mohamad, Nordin Ramli, Sigit P.W. Jarot. "Experimental Implementation of Dynamic Spectrum Access for Video Transmission using USRP,' International Conference on Computer and Communication Engineering, IEEE Computer Society, Malaysia, pp.228-233. July 2012 . 